\title{
Reduction of Constrained Mechanical Systems and Stability of Relative Equilibria
}

\section{Charles-Michel Marle}

Institut de Mathématiques de Jussieu, Université Pierre et Marie Curie, 4, place Jussieu, F-75252 Paris cedex 05, France. email: marle@mathp6.jussieu.fr

Received: 1 September 1994/in revised form: 15 April 1995

\begin{abstract}
A mechanical system with perfect constraints can be described, under some mild assumptions, as a constrained Hamiltonian system $(M, \Omega, H, D, W)$ : $(M, \Omega)$ (the phase space) is a symplectic manifold, $H$ (the Hamiltonian) a smooth function on $M, D$ (the constraint submanifold) a submanifold of $M$, and $W$ (the projection bundle) a vector sub-bundle of $T_{D} M$, the reduced tangent bundle along $D$. We prove that when these data satisfy some suitable conditions, the time evolution of the system is governed by a well defined differential equation on $D$. We define constrained Hamiltonian systems with symmetry, and prove a reduction theorem. Application of that theorem is illustrated on the example of a convex heavy body rolling without slipping on a horizontal plane. Two other simple examples show that constrained mechanical systems with symmetry may have an attractive (or repulsive) set of relative equilibria.
\end{abstract}

\section{Introduction}

Let $(M, \Omega)$ be a symplectic manifold on which a Lie group $G$ acts by a Hamiltonian action, with an equivariant momentum map $J$. Let $\mu$ be a regular (or weakly regular) value of $J$. Under some general assumptions, the very important concept of symplectic reduction, due to K. Meyer [24], J. Marsden and A. Weinstein [22], allows us to obtain from these data a new symplectic manifold $\left(P_{\mu}=J^{-1}(\mu) / G_{\mu}\right.$, $\Omega_{P}$ ), called the reduced symplectic manifold at $\mu$. Any smooth $G$-invariant Hamiltonian $H$ on $M$ induces a smooth reduced Hamiltonian $H_{P}$ on $P_{\mu}$, and the integral curves of the Hamiltonian vector field $X_{H}$ associated with $H$ contained in $J^{-1}(\mu)$ project onto the integral curves of the Hamiltonian vector field $X_{H_{P}}$ associated with $H_{P}$. In particular, relative equilibria of $X_{H}$ contained in $J^{-1}(\mu)$ project onto equilibria of $X_{H_{P}}$, i.e., onto points on $P_{\mu}$ where $X_{H_{P}}$ vanishes. Stability properties of these relative equilibria are closely related to stability properties of the corresponding equilibria in $P_{\mu}$.

Symplectic reduction plays an important part in symplectic geometry, in analytical mechanics and in mathematical physics. It has been extended to vari- 
ous more general situations, in which the symplectic manifold $(M, \Omega)$ is replaced by a Poisson manifold (J. Marsden and T. Ratiu [21]), a contact or a cosymplectic manifold (C. Albert [2]), a Jacobi manifold (K. Mikami [25], J. Nunes da Costa [27]). M. Kummer has given a nice description of the reduced symplectic manifold when $(M, \Omega)$ is the cotangent bundle of a principal $G$-bundle [14]. A. Weinstein has used the symplectic reduction procedure [39] in the minimal coupling construction introduced by S. Sternberg [30]. In this paper, in view of applications to mechanical systems with constraints, we introduce still another extension of the reduction concept. We were led to that extension by an attempt to understand the very remarkable stability properties of some nonholonomic mechanical systems such as the celtic stones, discussed a century ago by G.T. Walker $[36,37]$. The reader is referred to J. Walker [38] for a more recent and very clear (although nonmathematical) presentation of these properties. A different approach of the reduction procedure for nonholonomic mechanical systems was proposed earlier by L. Bates and J. Sniatycki [4].

The paper is organized as follows.

In Sect. 2, we present an intrinsic formulation of the dynamics of a constrained mechanical system. It is equivalent to the formulation of A.M. Vershik and L.D. Faddeev (see $[33,34,35]$ and the references therein), i.e., it leads to the same equations, but it is presented under a somewhat different manner more convenient for our particular application.

Section 3 considers an action of a Lie group on a constrained mechanical system, defines the reduction procedure and proves its main properties (Theorem 3.5).

Section 4 illustrates the reduction procedure with the classical example of a heavy convex body which rolls without slipping on a horizontal plane. If the body has a symmetry axis, and if the principal directions of curvature of the surface of the body at the lowest point on that axis differ from the principal directions of inertia, the stability properties of stationary rotations around that axis, set in a vertical position, may be quite complicated, very different from those of the stationary motions of a heavy body around a fixed point. The geometric setting presented here may help to understand these properties, which have been already investigated analytically by G.T. Walker almost a century ago [37], and more recently for example by A.V. Karapetian [9,10] and A.P. Markeev [17] in the linear approximation, M. Pascal for the asymptotic behaviour [28].

Finally Sect. 5 deals with two simple examples. The first one shows that a constrained mechanical system, even when the constraint is holonomic, may have two sets of relative equilibria, one of which is attractive and the other repulsive. An unconstrained conservative mechanical system, whose relative equilibria are neither attractive nor repulsive, cannot exhibit such a behaviour. The second example shows that a constrained Hamiltonian system, in the sense of Definition 3.1, may have an attractive equilibrium.

For the concept of Poisson manifold, the reader is referred to A. Lichnerowicz [16] and A. Weinstein [40]. The general definitions, notations and sign conventions about symplectic and Poisson manifolds are those of [15], not very different from those of Abraham and Marsden [1]. 


\section{The Dynamics of Constrained Dynamical Systems: An Intrinsic Formulation}

We present here an intrinsic formulation of the dynamics of a constrained mechanical system similar to that of A.M. Vershik and L.D. Faddeev [33,34,35]. This formulation is valid for very general constraints, which may be holonomic or nonholonomic, and eventually not linear in the velocities. For the sake of simplicity we present it for time-independent constraints, but its generalization to time dependent constraints, and even to active constraints $[18,19,7]$ should be easy. For constraints linear in the velocities, this formulation is equivalent to the classical one, which may be found for example in the treatise of Whittaker [42]. We do not follow here the other approach to nonholonomic constraints (the so called "vakonomic" approach) proposed by V.I. Arnold, V.V. Kozlov and A.I. Neishtadt [3] which leads to equations of motion not equivalent to the classical ones. A very thorough discussion of constrained systems may be found in Chapter 1 of the book by N. Woodhouse [43]. The reader is referred to S. Benenti [5], F. Cardin and G. Zanzotto [6], P. Dazord [7], J. Koiller [11], P.S. Krishnaprasad [12] and R. Yang [13,44], E. Massa and E. Pagani [23], for other approaches to the dynamics of constrained systems and applications.

2.1. Definition. $A$ constrained mechanical system is a triple $(Q, L, C)$, where $Q$ is a smooth manifold (the configuration space), $L: T Q \rightarrow \mathbf{R}$ a smooth function (the Lagrangian), and $C$ a smooth submanifold of the tangent bundle $T Q$ (the constraint submanifold of the system). The tangent bundle $T Q$ and the cotangent bundle $T^{*} Q$ of the configuration space are called the space of kinematical states and the phase space, respectively.

We shall denote by $p: T Q \rightarrow Q$ and $q: T^{*} Q \rightarrow Q$ the canonical projections.

2.2. The Lagrange differential. We recall that the Lagrangian $L$ defines a map $\Delta(L)$, called the Lagrange differential of $L$. This map is defined on the space $J^{2}(\mathbf{R}, 0 ; Q)$ of 2-jets at the origin of smooth parametrized curves in $Q$. It takes its values in the cotangent bundle $T^{*} Q$, and it is fibered over $Q$. This map can be defined in a coordinate-free manner $[8,29,31,32]$, but for simplicity we recall here only its expression in local coordinates. Let $\left(x^{1}, \ldots, x^{n}\right)$ be the local coordinates in a chart of $Q,\left(x^{1}, \ldots, x^{n}, v^{1}, \ldots, v^{n}\right),\left(x^{1}, \ldots, x^{n}, v^{1}, \ldots, v^{n}, a^{1}, \ldots, a^{n}\right)$ and $\left(x^{1}, \ldots, x^{n}, p_{1}, \ldots, p_{n}\right)$ the local coordinates in the associated charts of $T Q, J^{2}(\mathbf{R}, 0 ; Q)$ and $T^{*} Q$, respectively. Let $c: I \rightarrow Q$ be a smooth parametrized curve in $Q$, with $I$ an open interval in $\mathbf{R}$ such that $0 \in I$. We shall write

$$
x^{i}(t)=x^{l} \circ c(t), \quad v^{l}(t)=\frac{d}{d t}\left(x^{l} \circ c(t)\right), \quad a^{i}(t)=\frac{d^{2}}{d t^{2}}\left(x^{l} \circ c(t)\right)
$$

The local expression of the Lagrangian is a function $L$ of the local coordinates $\left(x^{1}, \ldots, x^{n}, v^{1}, \ldots, v^{n}\right)$ in $T Q$. Then the local coordinates $\left(x^{1}, \ldots, x^{n}, p_{1}, \ldots, p_{n}\right)$ of $\Delta(L)\left(j_{0}^{2} c\right)$ are

$$
\begin{gathered}
x^{l}=x^{l} \circ c(0) \\
p_{l}=\left.\left(\frac{d}{d s}\left(\frac{\partial L}{\partial v^{i}}(x(s), v(s))\right)-\frac{\partial L}{\partial x^{l}}(x(s), v(s))\right)\right|_{s=0}
\end{gathered}
$$


where $x(s)$ and $v(s)$ stand for $\left(x^{1}(s), \ldots, x^{n}(s)\right)$ and $\left(v^{1}(s), \ldots, v^{n}(s)\right)$, respectively. As is well known, the $x^{i}$ and $p_{l}$ given by these formulae depend only on the local coordinates $x^{i}(0), v^{l}(0)$ and $a^{i}(0), i \leqq i \leqq n$, of the 2-jet $j_{0}^{2} c$ of $c$ at the origin.

2.3. The Lagrange Equations of Motion. In the Lagrangian formalism, the equations of motion of the constrained mechanical system $(Q, L, C)$ can be written as

$$
\Delta(L)=f,
$$

where $\Delta(L)$ is the Lagrange differential of $L$ discussed in Section 2.2. On the right hand side, $f$ is the constraint force. It is in general unknown, but must satisfy some conditions, which depend on the physical properties of the constraint. These conditions will be indicated in Section 2.6 for a particular class of constraints (the so called perfect constraints). A motion of the system, i.e., a solution of (1), is a smooth curve $c: I \rightarrow C$, where $I$ is an open interval in $\mathbf{R}$, such that, for each $t \in I, \frac{d c(t)}{d t}$ lies in $C$ and the constraint force $f(t)$, defined by

$$
\Delta(L)\left(j^{2} c(t)\right)=f(t),
$$

satisfies these conditions.

2.4. The Legendre Transformation. We recall that, associated with the Lagrangian $L$, there is a map $\mathscr{L}: T Q \rightarrow T^{*} Q$, called the Legendre transformation. This map is such that, for each $v \in T Q, \mathscr{L}(v) \in T_{p(v)}^{*} Q$ is the differential at $v$ of the restriction of $L$ to the tangent space $T_{p(v)} Q$.

2.5. Definitions. Let $(Q, L, C)$ be a constrained mechanical system.

1. The constraint $C$ is said to be regular if there exists a submanifold $Q_{1}$ of $Q$ such that the constraint submanifold $C$ is contained in $T Q_{1}$ (considered as a submanifold of $T Q$ ) and that the restriction to $C$ of the canonical projection $p: T Q \rightarrow Q$ is a submersion of $C$ onto $Q_{1}$.

2. The Lagrangian $L$ is said to be regular if the Legendre transformation $\mathscr{L}$ : $T Q \rightarrow T^{*} Q$ associated with $L$ is a diffeomorphism.

3. The constrained system $(Q, L, C)$ is said to be regular if the constraint and the Lagrangian are both regular.

\subsection{Examples and Remarks. Let $(Q, L, C)$ be a constrained mechanical system.}

1. The constraint is said to be purely geometric if there exists a submanifold $Q_{1}$ of $Q$ such that $C=T Q_{1}$. A purely geometric constraint is automatically regular. Such a constraint can be eliminated by choosing $Q_{1}$ as configuration space instead of $Q$. 2. The constraint is said to be purely kinematic if $p(C)=Q$, i.e., if every point of $Q$ is a possible configuration of the system. Such a constraint is regular if the restriction to $C$ of the canonical projection $p: T Q \rightarrow Q$ is a submersion of $C$ onto $Q$.

3. Very often, the constraint submanifold $C$ is a vector sub-bundle of $T Q$. Such a constraint is purely kinematic and regular. When in addition the sub-bundle $C$ is completely integrable, we shall say that the constraint is holonomic. In such a case $C$ defines a foliation of $Q$. If the configuration of the system is in a leaf $Q_{1}$ of that foliation at a particular time $t_{0}$, it is in $Q_{1}$ for all times. The constraint submanifold $C$ may then be replaced by $T Q_{1}$, which is now a purely geometric 
constraint. However, this procedure is suitable only to deal with the motions of the system for which the configuration is in the particular leaf $Q_{1}$ of the foliation of $Q$ determined by $C$; it is not suitable to deal with stability properties, since motions of the system for which the configuration is in leaves of that foliation near $Q_{1}$, but distinct from $Q_{1}$, must be taken into account.

4. When the constraint is regular, by taking $Q_{1}=p(C)$ instead of $Q$ as configuration space, we can replace the system by a completely equivalent one in which the constraint is purely kinematic and regular. However, in many examples, such as the one dealt with in Sect. 4, it is more convenient to keep as configuration space a manifold $Q$ strictly larger than the set $Q_{1}=p(C)$ of all the possible configurations compatible with the constraint.

2.7. Admissible Infinitesimal Virtual Displacements. We assume now that $(Q, L, C)$ is a regular constrained mechanical system. Let $v \in C$ be any point of the constraint submanifold, and $x=p(v) \in Q$. Since $\left.p\right|_{C}$ is a submersion of $C$ onto the submanifold $Q_{1}=p(C)$ of $Q, C_{x}=C \cap T_{x} Q$ is a submanifold of $T_{x} Q_{1}$. Therefore the space $T_{v} C_{x}$, tangent at $v$ to a submanifold of a vector space, can be considered as a vector subspace of that vector space $T_{x} Q_{1}$, which is itself a vector subspace of $T_{x} Q$. The vector space $T_{v} C_{x}$ is called the space of admissible infinitesimal virtual displacements at $v$. Its annihilator $V_{v}=\left(T_{v} C_{x}\right)^{0}$ is the vector subspace of all $\eta \in T_{x}^{*} Q$ such that $\langle\eta, w\rangle=0$ for all $w \in T_{v} C_{x}$. We are led to the following definition.

2.8. Definition. The constraint is said to be perfect if, for each $v \in C, x=p(v) \in$ $Q$, the constraint force $f$ takes its value in the annihilator $V_{v}=\left(T_{v} C_{x}\right)^{0}$ of the space of admissible infinitesimal virtual displacements $T_{v} C_{x}$ when the kinematical state of the system is $v$.

The above definition is in agreement with the principle (called the d'AlembertLagrange principle in Arnold, Kozlov and Neishtadt [3]) which states that when a constraint is perfect, the virtual work of the constraint force for any infinitesimal virtual displacement compatible with the constraint vanishes.

2.9. The Map $\lambda$. For any vector bundle $(E, \pi, Q)$ over the base manifold $Q$, let $E \times{ }_{Q} E$ be the fibered product of $E$ with itself over $Q$, and $\lambda: E \times_{Q} E \rightarrow T E$ be the map such that, for any $x \in Q, v$ and $w \in E_{x}, \lambda(v, w)$ is the vector equal to $w$, tangent at $v$ to the fiber $E_{x}$. Since $E_{x}$ is a vector space, we have identified it with its tangent space at $v, T_{v} E_{x}$. The map $\lambda$ is an injective vector bundle map ( $E \times_{Q} E$ being considered as a vector bundle over $E$, with canonical projection $(v, w) \mapsto w)$, which maps $E \times{ }_{Q} E$ onto the vertical bundle $V E=\operatorname{ker}(T \pi)$. In the following definition we use the map $\lambda$ for the cotangent bundle $\left(T^{*} Q, q, Q\right)$.

2.10. Definitions. Let us assume that the constrained mechanical system $(Q, L, C)$ is regular.

1. The image $D=\mathscr{L}(C)$ of the constraint submanifold $C$ by the Legendre transformation will be called the Hamiltonian constraint submanifold.

2. For any $p \in D$, the vector subspace $W_{p}$ of the tangent space $T_{p}\left(T^{*} Q\right)$, defined by

$$
W_{p}=\left\{\lambda(p, \eta) \mid \eta \in V_{\mathscr{L}^{-1}(p)}\right\}
$$


will be called the projection space at $p$. The union

$$
W=\bigcup_{p \in D} W_{p}
$$

of all the projection spaces at points of $D$ is a vector sub-bundle of $T_{D}\left(T^{*} Q\right)$, which will be called the projection bundle.

We observe that $W$ is a subset of the vertical bundle $\operatorname{ker}(T q)$.

2.11. Definition. Let $v \in T Q$ be a kinematic state, $x=p(v) \in Q$, and $L_{x}=\left.L\right|_{T_{x} Q}$. The Lagrangian $L$ is said to be normal at $v$ if the matrix

$$
\left(\frac{\partial^{2} L_{x}}{\partial v^{i} \partial v^{j}}(v)\right), \quad 1 \leqq i, j \leqq n,
$$

of second partial derivatives of $L_{x}$ at $v$, with respect to the coordinates $v^{1}, \ldots, v^{n}$ in $T_{x} Q$ associated with a basis of that vector space, is positive definite.

This definition has indeed an intrinsic meaning, since the positive definiteness of that matrix does not depend on the choice of the basis of $T_{x} Q$.

2.12. Proposition. We assume that the constrained mechanical system $(Q, L, C)$ is regular and that the Lagrangian $L$ is normal at every point of the constraint submanifold $C$. Then the sum of the vector sub-bundles $T D$ and $W$ of $T_{D}\left(T^{*} Q\right)$ (restriction of the tangent bundle $T\left(T^{*} Q\right)$ to the Hamiltonian constraint submanifold $D$ ) is a direct sum, and

$$
T D \oplus W=\left(T_{D} q\right)^{-1}\left(T Q_{1}\right)
$$

where $Q_{1}=p(C)$ is the submanifold of $Q$ introduced in Definition 2.5.

Proof. Let $\operatorname{dim} Q=n, \operatorname{dim} Q_{1}=m \leqq n$. Since the property to be proven is local, we can use a chart of $Q$ adapted to the submanifold $Q_{1}$, with local coordinates $(x)=\left(x^{1}, \ldots, x^{n}\right)$. In that chart $Q_{1}$ is locally defined by the equations

$$
x_{i}=0 \text { for } m+1 \leqq i \leqq n .
$$

Let $(x, v)=\left(x^{1}, \ldots, x^{n}, v^{1}, \ldots, v^{n}\right)$ and $(x, p)=\left(x^{1}, \ldots, x^{n}, p_{1}, \ldots, p_{n}\right)$ be the local coordinates in the associated charts of $T Q$ and $T^{*} Q$ respectively. The constraint submanifold $C$ is contained in $T Q_{1}$, therefore it can be locally defined by the $2(n-m)+k$ equations

$$
x^{i}=0 \quad \text { and } \quad v^{i}=0 \quad \text { for } m+1 \leqq i \leqq n, \quad \Phi^{\alpha}(x, v)=0, \quad 1 \leqq \alpha \leqq k .
$$

Since $\left.q\right|_{C}$ is a submersion of $C$ onto $Q_{1}$, the functions $\Phi^{\alpha}$ can be chosen in such a way that the matrix

$$
\left(\frac{\partial \Phi^{\alpha}}{\partial v^{j}}\right), \quad 1 \leqq \alpha \leqq k, \quad 1 \leqq j \leqq m,
$$

is of rank $k$ at all points of $C$. The tangent space at $(x, v)$ to the constraint submanifold $C$ is the set of all vectors

$$
\sum_{i=1}^{m}\left(A^{i} \frac{\partial}{\partial x^{i}}+B^{i} \frac{\partial}{\partial v^{i}}\right)
$$


whose components $A^{l}$ and $B^{i}$ satisfy

$$
\sum_{i=1}^{m}\left(A^{i} \frac{\partial \Phi^{\alpha}}{\partial x^{l}}+B^{i} \frac{\partial \Phi^{\alpha}}{\partial v^{l}}\right)=0, \quad 1 \leqq \alpha \leqq k .
$$

Since $D=\mathscr{L}(C)$, and since $\mathscr{L}$ maps $(x, v) \in C$ onto $(x, p) \in D$, with

$$
p_{l}=\frac{\partial L(x, v)}{\partial v^{l}},
$$

the tangent space at $(x, p)$ to the Hamiltonian constraint submanifold $D$ is the set of all vectors

$$
\sum_{i=1}^{m}\left(A^{i}\left(\frac{\partial}{\partial x^{i}}+\sum_{j=1}^{n} \frac{\partial^{2} L}{\partial v^{j} \partial x^{i}} \frac{\partial}{\partial p_{J}}\right)+B^{l} \sum_{j=1}^{n} \frac{\partial^{2} L}{\partial v^{j} \partial v^{i}} \frac{\partial}{\partial p_{J}}\right)
$$

with $A^{l}$ and $B^{i}$ satisfying (4).

The fiber $W_{(x, p)}$ of the projection bundle at point $(x, p) \in D$ is generated by the $n-m+k$ vectors

$$
\frac{\partial}{\partial p_{l}} \text { for } m+1 \leqq i \leqq n \text { and } \sum_{i=1}^{m} \frac{\partial \Phi^{\alpha}}{\partial v^{i}} \frac{\partial}{\partial p_{i}} \text { for } 1 \leqq \alpha \leqq k .
$$

The fiber at $(x, p) \in D$ of $\left(T_{D} q\right)^{-1}\left(T Q_{1}\right)$ is generated by the vectors

$$
\frac{\partial}{\partial x^{i}} \text { for } 1 \leqq i \leqq m \text { and } \frac{\partial}{\partial p_{j}} \text { for } 1 \leqq j \leqq n .
$$

That fiber is of dimension $n+m$, and it contains $T_{(x, p)} D$ and $W_{(x, p)}$, whose dimensions are $2 m-k$ and $n-m+k$, respectively. Therefore we have only to prove that $W_{(x, p)} \cap T_{(x, p)} D=\{0\}$, i.e., that the only $\lambda_{x}(1 \leqq \alpha \leqq k), \mu_{j}(m+1 \leqq j \leqq n)$, $A^{i}$ and $B^{i}(1 \leqq i \leqq m)$ which satisfy (4) and

$$
\begin{aligned}
& \sum_{x=1}^{k}\left(\lambda_{\alpha} \sum_{j=1}^{n} \frac{\partial \Phi^{\alpha}}{\partial v^{j}} \frac{\partial}{\partial p_{J}}\right)+\sum_{J=m+1}^{n} \mu_{j} \frac{\partial}{\partial p_{J}} \\
& \quad=\sum_{i=1}^{m} A^{i} \frac{\partial}{\partial x^{i}}+\sum_{i=1}^{m} \sum_{j=1}^{n}\left(A^{i} \frac{\partial^{2} L}{\partial v^{J} \partial x^{i}}+B^{i} \frac{\partial^{2} L}{\partial v^{J} \partial v^{i}}\right) \frac{\partial}{\partial p_{j}}
\end{aligned}
$$

vanish identically. But (5) implies

$$
A^{l}=0 \quad \text { for } 1 \leqq i \leqq m,
$$

and

$$
\sum_{\alpha=1}^{k} \lambda_{\alpha} \frac{\partial \Phi^{\alpha}}{\partial v^{\prime}}+\mu_{j}=\sum_{i=1}^{m} B^{i} \frac{\partial^{2} L}{\partial v^{j} \partial v^{i}} \quad \text { for } 1 \leqq j \leqq n,
$$

where we have set $\mu_{j}=0$ for $1 \leqq j \leqq m$. Since the $A^{l}$ vanish, (4) implies

$$
\sum_{l=1}^{m} B^{\prime} \frac{\partial \Phi^{\alpha}}{\partial v^{l}}=0
$$


Multiplying Eq. (6) by $B^{j}$, summing up over $j$ for $1 \leqq j \leqq m$ and using (7), we obtain

$$
\sum_{i=1}^{m} \sum_{j=1}^{m} B^{i} B^{j} \frac{\partial^{2} L}{\partial v^{i} \partial v^{j}}=0 .
$$

Since $L$ is normal at all points of $C$, the above equality implies that $B^{i}=0$ for all $i, 1 \leqq i \leqq m$. Then, since the matrix (3) is of rank $k$ at all points of $C$, (6) implies that $\lambda^{\alpha}=0$ for all $\alpha, 1 \leqq \alpha \leqq k$, and that $\mu_{j}=0$ for all $j, m+1 \leqq j \leqq n$.

2.13. Remark. The property of $L$ used in the proof is slightly less restrictive than the property stated in Definition 2.11, since the indices $i$ and $j$ in Eq. (8) satisfy $1 \leqq i, j \leqq m$, instead of $1 \leqq i, j \leqq n$. Therefore it should be possible to weaken a little Definition 2.11 .

2.14. The Symplectic Structure of $T^{*} Q$ and the Hamiltonian Vector field $X_{H}$. The assumptions are the same as in Proposition 2.10. We define the Hamiltonian $H: T^{*} Q \rightarrow \mathbf{R}$ of the system by setting

$$
H=(i(Z) d L-L) \circ \mathscr{L}^{-1},
$$

where $Z$ is the Liouville vector field on $T Q$. In local coordinates

$$
Z=\sum_{l=1}^{n} v^{i} \frac{\partial}{\partial v^{l}}, \quad H(x, p)=\sum_{i=1}^{n} v^{i} p_{i}-L(x, v),
$$

where, in the right-hand side, the local coordinates $(x, v)$ in $T Q$ must be expressed in terms of the local coordinates $(x, p)$ in $T^{*} Q$, by means of the diffeomorphism $\mathscr{L}^{-1}$.

Let $\alpha$ be the Liouville 1-form and $\Omega=d \alpha$ the canonical symplectic 2-form on $T^{*} Q$. In local coordinates,

$$
\alpha=\sum_{i=1}^{n} p_{i} d x^{i}, \quad \Omega=\sum_{i=1}^{n} d p_{i} \wedge d x^{i} .
$$

Let $X_{H}$ be the Hamiltonian vector field associated with $H$, i.e., the vector field such that

$$
i\left(X_{H}\right) \Omega=-d H .
$$

2.15. Proposition. Under the assumptions of Proposition 2.12, the restriction $\left.X_{H}\right|_{D}$ of the Hamiltonian vector field to the Hamiltonian constraint submanifold $D$ splits into a sum

$$
\left.X_{H}\right|_{D}=X_{D}+X_{W},
$$

where $X_{D}$ is a smooth vector field tangent to $D$ and $X_{W}$ a smooth section of the projection bundle $W$. The vector field $X_{D}$ will be called the constrained Hamiltonian vector field, and the opposite $-X_{W}$ of $X_{W}$, the constraint force field.

Proof. In view of Proposition 2.12, it is enough to prove that $\left.X_{H}\right|_{D}$ is a section of $T D \oplus W=\left(T_{D} q\right)^{-1}\left(T Q_{1}\right)$, or equivalently that, for any $z \in D, T q\left(X_{H}(z)\right)$ is tangent to the submanifold $Q_{1}$. As in the proof of Proposition 2.12, we use a chart of $Q$ adapted to the submanifold $Q_{1}$, and the associated charts of $T Q$ and $T^{*} Q$. The vector field $X_{H}$ may be written as

$$
X_{H}=\sum_{l=1}^{n}\left(\frac{\partial H}{\partial p_{l}} \frac{\partial}{\partial x^{i}}-\frac{\partial H}{\partial x^{i}} \frac{\partial}{\partial p_{l}}\right) .
$$


Let $(x, v)$ be a point of $C$. Since $C \subset T Q_{1}$, we have $v^{l}=0$ for $m+1 \leqq i \leqq n$. Let $(x, p) \in D$ be the image of $(x, v)$ by the Legendre transformation. We have, for all $i, 1 \leqq i \leqq n$,

$$
\frac{\partial H}{\partial p_{l}}=v^{l}
$$

therefore

$$
\frac{\partial H}{\partial p_{l}}=0 \text { for } m+1 \leqq i \leqq n
$$

This shows that the projection of $\left.X_{H}\right|_{D}$ on $Q$ is tangent to $Q_{1}$.

2.16. Theorem. We assume that the constrained mechanical system $(Q, L, C)$ is regular, that the Lagrangian $L$ is normal at every point of the constraint submanifold $C$, and that the constraint is perfect. A parametrized curve $c: I \rightarrow Q$ (with $I$ an open interval in $\mathbf{R})$ is a motion of the system if and only if its canonical lift $\widetilde{c}$ to the cotangent bundle, defined by

$$
\widetilde{c}=\mathscr{L} \circ \frac{d c}{d t},
$$

is an integral curve of the constrained Hamiltonian vector field $X_{D}$. When such is the case, the constraint force $f(t)$ at each time $t \in I$ is the unique element in $T_{c(t)}^{*} Q$ such that

$$
\lambda(\widetilde{c}(t), f(t))=-X_{W}(\widetilde{c}(t)) .
$$

Proof. In local coordinates $\left(x^{i}, v^{i}\right)$ in $T Q,\left(x^{i}, p_{l}\right)$ in $T^{*} Q$, related by the Legendre transformation $\mathscr{L}$, it is well known that the constrained Lagrange equations

$$
\frac{d}{d t} \frac{\partial L}{\partial v^{l}}-\frac{\partial L}{\partial x^{l}}=f_{l}, \quad 1 \leqq i \leqq n
$$

are equivalent to the constrained Hamilton equations

$$
\begin{aligned}
& \frac{d x^{i}}{d t}=\frac{\partial H}{\partial p_{i}}, \\
& \frac{d p_{i}}{d t}=-\frac{\partial H}{\partial x^{i}}+f_{i} .
\end{aligned}
$$

Let us assume that $t \mapsto c(t)$ is a motion of the system. Its canonical lift $t \mapsto \widetilde{c}(t)$ to the cotangent bundle lies in the Hamiltonian constraint submanifold $D$. Therefore, for each $t \in I$, the vector

$$
\sum_{i=1}^{n}\left(\frac{\partial H}{\partial p_{i}} \frac{\partial}{\partial x^{i}}+\left(-\frac{\partial H}{\partial x^{i}}+f_{l}\right) \frac{\partial}{\partial p_{l}}\right)(\widetilde{c}(t))=X_{H}(\widetilde{c}(t))+\left(\sum_{l=1}^{n} f_{i} \frac{\partial}{\partial p_{i}}\right)(\widetilde{c}(t))
$$

is tangent to $D$. Since the constraint is perfect, the second term in the right-hand side of the above expression is in $W_{\check{c}(t)}$, and we have

$$
\begin{aligned}
& X_{D}(\tilde{c}(t))=\sum_{i=1}^{n}\left(\frac{\partial H}{\partial p_{i}} \frac{\partial}{\partial x^{i}}+\left(-\frac{\partial H}{\partial x^{i}}+f_{i}\right) \frac{\partial}{\partial p_{i}}\right)(\widetilde{c}(t)), \\
& X_{W}(\widetilde{c}(t))=-\left(\sum_{i=1}^{n} f_{i} \frac{\partial}{\partial p_{i}}\right)(\widetilde{c}(t)) .
\end{aligned}
$$


This proves that $t \mapsto \widetilde{c}(t)$ is an integral curve of $X_{D}$ and that, for each $t \in I$, $\lambda(\widetilde{c}(t), f(t))=-X_{W}(\widetilde{c}(t))$.

Conversely, if $t \mapsto \widetilde{c}(t)$ is an integral curve of $X_{D}$, the constrained Hamilton equations above are satisfied, with

$$
\sum_{i=1}^{n} f_{i} \frac{\partial}{\partial p_{i}}=-X_{W}
$$

Since $X_{W}$ is a section of $W$, the condition which states that the constraint is perfect is satisfied and the projection on $Q$ of the curve $t \mapsto \widetilde{c}(t)$ is a motion of the system.

\subsection{Remarks.}

1. Contrary to what happens for unconstrained, time independent systems, the Hamiltonian $H$ may not be a first integral of $X_{D}$, i.e., may not remain constant during a motion of the system. This property is due to the fact that even when the constraint is time independent, it may give energy to (or withdraw energy from) the system. However, when the constraint submanifold $C$ is tangent to the Liouville vector field of $T Q$ (in particular, when $C$ is a vector sub-bundle of $T Q$ ), one can prove that $H$ is a first integral of $X_{D}$.

2. In [7], P. Dazord introduces a formalism for constrained mechanical systems different from that used here. In his formalism, the geometric properties of the constraint are described by a submersion $f$ of the configuration manifold $Q$ onto another manifold $S$, and a map $Y: T Q \rightarrow T S$, fibered over $f$, eventually nonlinear on the fibers. A parametrized curve $t \mapsto x(t)$ in $Q$ is said to be compatible with the constraint when it satisfies

$$
\frac{d}{d t} f(x(t))=Y\left(\frac{d x(t)}{d t}\right) .
$$

In other words, the subset $C$ of $T Q$ of kinematical states compatible with the constraint is

$$
C=\{v \in T Q \mid(T f-Y)(v)=0\} .
$$

Dazord does not assume that $C$ is a submanifold of $T Q$. He says that the constraint is holonomic when $Y=0$, almost holonomic when $Y$ factors through $f \circ p: T Q \rightarrow$ $S$, semi-holonomic when $Y$ factors through $p: T Q \rightarrow Q$. In order to describe the mechanical properties of the constraint, he introduces a vector sub-bundle $F$ of $p^{*}(T Q)$ (the vector bundle over $T Q$ inverse image of the tangent bundle $T Q$ by the map $p: T Q \rightarrow Q$ ). The sub-bundle $F$ yields the space of infinitesimal virtual displacements for which the work of the constraint force vanishes. Dazord says that the constraint is perfect when

$$
F=\left\{(v, w) \in p^{*}(T Q) \equiv T Q \oplus T Q \mid w \in \operatorname{ker}(T f)\right\} .
$$

Observe that $Y$ does not play any part in the definition of perfect constraints. For semi-holonomic constraints (in Dazord's language), and a fortiori for almost holonomic or holonomic constraints, one can easily see that Dazord's definition of perfect constraints is equivalent to our Definition 2.8, and that Dazord's formalism and ours lead to the same equations. But for more general constraints, the two formalisms are not equivalent, even in the definition of perfect constraints. Of course, the formalism developed here deals only with passive constraints, while Dazord's 
formalism (which extends some ideas introduced in [18 and 19] for geometric constraints) can include some active constraints.

\section{Lie Group Actions on Constrained Systems, Reduction and Relative Equilibria}

In this section we consider the action of a Lie group on a constrained mechanical system. We define relative equilibria (also called stationary motions) of the system. We introduce a reduction procedure which will allow us to obtain a reduced constrained system, and we use that reduced system for discussing the stability of relative equilibria. The reader is referred to J.E. Marsden [20] for a thorough discussion of various kinds of reduction and many applications, and to R. Montgomery, J.E. Marsden and T. Ratiu [26] for the determination of the Poisson structure on the quotient of the cotangent bundle to a principal $G$-bundle.

For the needs of the reduction procedure to be defined, we must first introduce a slight generalization of the concept of constrained Hamiltonian system, in which the phase space will be a general Poisson manifold, instead of a cotangent bundle equipped with its canonical symplectic structure and the associated Poisson structure.

3.1. Definition. $A$ constrained Hamiltonian system is a 5-tuple $(M, \Lambda, H, D, W)$, in which

- $(M, \Lambda)$ is a Poisson manifold (the phase space),

- H:M $\rightarrow \mathbf{R}$ is a smooth function (the Hamiltonian),

- $D$ is a submanifold of $M$ (the Hamiltonian constraint submanifold),

- $W$ is a vector sub-bundle of $T_{D} M$ (the projection bundle) such that $T D \cap W=$ $\{0\}$ and that the Hamiltonian vector field $X_{H}=\Lambda^{\sharp}(d H)$, restricted to the submanifold $D$, is a section of $T D \oplus W$.

We set

$$
\left.X_{H}\right|_{D}=X_{D}+X_{W}
$$

where $X_{D}$ is a smooth vector field on $D$ (called the constrained Hamiltonian vector field), and $X_{W}$ a smooth section of the projection bundle $W$ (its opposite $-X_{W}$ is called the constraint force field). Integral curves of $X_{D}$ are called motions of the system.

We observe that the property which states that $T D \cap W=\{0\}$ and that $\left.X_{H}\right|_{D}$ is a section of $T D \oplus W$, in Definition 3.1, is equivalent to the pairing condition of Dazord [7].

3.2. Definition. $A$ symmetry of the constrained Hamiltonian system $(M, \Lambda, H, D, W)$ is a left action $\Phi: G \times M \rightarrow M$ of a Lie group $G$ on the phase space $M$, which leaves invariant the Poisson tensor $\Lambda$, the Hamiltonian $H$, the Hamiltonian constraint submanifold $D$, and the projection bundle $W$.

3.3. Remarks. The symmetry $\Phi$ is, in many examples, a Hamiltonian action, i.e., an action such that the infinitesimal generator of the restriction of $\Phi$ to every oneparameter subgroup of $G$ is a Hamiltonian vector field. In such a case, there exists a momentum map $J: M \rightarrow \mathscr{G}^{*}$, where $\mathscr{G}^{*}$ is the dual of the Lie algebra $\mathscr{G}$ of $G$, such that, for any $X \in \mathscr{G}$, the infinitesimal generator of the restriction of $\Phi$ to $\{\exp (t X) \mid t \in \mathbf{R}\}$ is the Hamiltonian vector field $\Lambda^{\sharp}(d\langle J, X\rangle)$. For classical mechanical systems with constraints discussed in Sect. 2, the phase space $M$ is a cotangent 
bundle $T^{*} Q$, its Poisson structure is the structure associated with its canonical symplectic structure $\Omega=d \alpha$, and the action $\Phi$ is, in most cases, the canonical lift to $T^{*} Q$ of an action of a Lie group $G$ on the configuration space $Q$. Such an action leaves invariant the Liouville 1 -form $\alpha$ of $T^{*} Q$, and therefore is Hamiltonian, with an $\mathrm{Ad}^{*}$-equivariant momentum map. However, for constrained Hamiltonian systems, the momentum map does not play a part as important as for unconstrained systems, because in general it does not remain constant during a motion of the system: Noether's theorem does not apply. Moreover, a Hamiltonian action of a connected Lie group on a Poisson manifold leaves invariant every symplectic leaf of that manifold. The symmetries of constrained Hamiltonian systems obtained by reduction very often do not leave invariant the symplectic leaves of the phase space, but instead they map every symplectic leaf onto another symplectic leaf. For these reasons, in Definition 3.2, the symmetry $\Phi$ is a Poisson action which may not be Hamiltonian.

3.4. Definition. Let $(M, \Lambda, H, D, W)$ be a constrained Hamiltonian system with a symmetry $\Phi: G \times M \rightarrow M$. A relative equilibrium (also called stationary motion) of the system is a motion $c: \mathbf{R} \rightarrow D$ of the system, i.e., an integral curve of the constrained Hamiltonian vector field $X_{D}$, which is also an orbit, under the action $\Phi$, of a one-parameter subgroup of $G$.

3.5. Theorem. Let $(M, \Lambda, H, D, W)$ be a constrained Hamiltonian system with a symmetry $\Phi: G \times M \rightarrow M$. We assume that the set $\widehat{M}$ of orbits of the action $\Phi$ has a smooth manifold structure such that the canonical projection $\pi: M \rightarrow \widehat{M}$ is a submersion. Then:

1. $\widehat{D}=\pi(D)$ is a submanifold of $\widehat{M}$, and the image by $\pi$ of the bundle $W$ is a vector sub-bundle $\widehat{W}$ of $T_{\hat{D}} \widehat{M}$, such that $T \widehat{D} \cap \widehat{W}=\{0\}$. Moreover, there exists on $\widehat{M}$ a unique Poisson structure $\widehat{\Lambda}$ such that $\pi$ is a Poisson map, and a unique smooth function $\widehat{H}: \widehat{M} \rightarrow \mathbf{R}$, such that $H=\widehat{H} \circ \pi$.

2. $(\widehat{M}, \widehat{\Lambda}, \widehat{H}, \widehat{D}, \widehat{W})$ is a constrained Hamiltonian system, which will be called the reduced constrained system associated with $(M, \Lambda, H, D, W)$ and the symmetry $\Phi$.

3. For each motion $c$ of the constrained Hamiltonian system $(M, \Lambda, H, D, W)$, $\widehat{c}=\pi \circ c$ is a motion of the reduced system $(\widehat{M}, \widehat{\Lambda}, \widehat{H}, \widehat{D}, \widehat{W})$.

4. $A$ motion $c$ of $(M, \Lambda, H, D, W)$ is a relative equilibrium if and only if $\widehat{c}=\pi \circ c$ is constant, i.e., is an equilibrium point of the reduced system.

\section{Proof.}

1. For any point $\widehat{a} \in \widehat{D}$, there exists a smooth section $s: \widehat{U} \rightarrow M$ of the submersion $\pi$, defined on an open neighbourhood $\widehat{U}$ of $\widehat{a}$ in $\widehat{M}$. Then $s$ is a diffeomorphism of $\widehat{U}$ onto the submanifold $U=s(\widehat{U})$ of $M$. Moreover, $U$ intersects $D$ transversally, because for each point $x \in U \cap D, T_{x} D$ contains $\operatorname{ker}\left(T_{x} \pi\right)$ which is complementary to $T_{x} U$ in $T_{x} M$. Therefore $U \cap D$ is a submanifold of $U$. Since $D$ is $\Phi$-invariant, $s$ maps $\widehat{U} \cap \widehat{D}$ onto $U \cap D$, which proves that $\widehat{U} \cap \widehat{D}$ is a submanifold of $\widehat{U}$. We conclude that $\widehat{D}$ is a submanifold of $\widehat{M}$.

Let $\widehat{x}$ be a point of $\widehat{D}$, and $x$ be a point such that $\pi(x)=\widehat{x}$. By using the invariance of $W$, we see that $T_{x} \pi\left(W_{x}\right)$ does not depend on the choice of $x \in \pi^{-1}(x)$. Therefore we can set $\widehat{W}_{\hat{x}}=T_{x} \pi\left(W_{x}\right)$. We have $T_{x} D \cap W_{x}=\{0\}$ and $\operatorname{ker}\left(T_{x} \pi\right) \subset$ $T_{x} D$; therefore the restriction of $T_{x} \pi$ to $W_{x}$ is an isomorphism of $W_{x}$ onto $\widehat{W}_{\hat{x}}$, which 
proves that $\widehat{W}=\bigcup_{\hat{x} \in \hat{D}} \widehat{W}_{\hat{x}}$ is vector sub-bundle of $T_{\hat{D}} \widehat{M}$. Moreover, $T_{x} \pi\left(T_{x} D\right)=$ $T_{\hat{x}} \widehat{D} \cap \widehat{W}_{\hat{x}}=\{0\}$.

By assumption, $\Phi$ leaves $\Lambda$ invariant. Therefore the Poisson bracket of two $\Phi$ invariant smooth functions on $M$ is a $\Phi$-invariant function. This implies [15, Chapter III, Proposition 9.4] that there exists a unique Poisson structure $\widehat{\Lambda}$ on $\widehat{W}$ such that $\pi$ is a Poisson map.

Smooth functions on $\widehat{M}$ are in one-to-one correspondence with $\Phi$-invariant smooth functions on $M$. Since $H$ is $\Phi$-invariant, there exists a unique smooth function $\widehat{H}$ on $\widehat{M}$ such that $H=\widehat{H} \circ \pi$.

2. Since $\pi$ is a Poisson map, the Hamiltonian vector field $X_{H}$ on the Poisson manifold $(M, \Lambda)$ projects onto the hamiltonian vector field $X_{\hat{H}}$ on the Poisson manifold $(\widehat{M}, \widehat{\Lambda})$. Therefore $\left.X_{\hat{H}}\right|_{\hat{D}}$ is a section of $T \widehat{D} \oplus \widehat{W}$, and $(\widehat{M}, \widehat{\Lambda}, \widehat{H}, \widehat{D}, \widehat{W})$ is a constrained Hamiltonian system.

3. The two terms $X_{D}$ and $X_{W}$ of the restriction of $X_{H}$ to $D$ project onto $X_{\hat{D}}$ and $X_{\hat{W}}$, respectively. Therefore, for each motion $c$ of $(M, \Lambda, H, D, W), \widehat{c}=\pi \circ c$ is a motion of the reduced system $(\widehat{M}, \widehat{A}, \widehat{H}, \widehat{D}, \widehat{W})$.

4. Let $c$ be a relative equilibrium of $(M, \Lambda, H, D, W)$. Then by the very definition of a relative equilibrium, $\pi \circ c$ is constant, i.e, is an equilibrium point of the reduced system. Conversely, let $c: I \rightarrow M$ be a motion of $(M, \Lambda, H, D, W)$ such that $\pi \circ c$ is constant. Let $t_{0} \in I$, and $x_{0}=x\left(t_{0}\right)$. For any $t \in I, x(t)$ is an element of the orbit $\Phi\left(G, x_{0}\right)$, which may be identified with an homogeneous space of $G$. Therefore there exists an element $X \in \mathscr{G}$ such that

$$
X_{D}\left(x_{0}\right)=\left.\frac{d}{d s} \Phi\left(\exp (s X), x_{0}\right)\right|_{s=0} .
$$

Using the $\Phi$-invariance of the constrained Hamiltonian vector field $X_{D}$, we see that the parametrized curve $t \mapsto \Phi\left(\exp \left(t-t_{0}\right) X, x_{0}\right)$ is an integral curve of $X_{D}$ which takes the value $x_{0}$ for $t=t_{0}$. By unicity of maximal integral curves, that parametrized curve is equal to $c$.

\section{The Rolling Stone}

4.1. Configuration, Kinematical States, Phase Space and Hamiltonian. Let us consider a solid heavy body, bounded by a smooth, strictly convex surface, supported by a fixed solid horizontal plane. We assume that during its motion, the body remains in contact with the plane, that the friction at the contact point prevents the body from slipping, and that the corresponding constraint is perfect.

We first define the configuration space of the system without constraint. Let $E$ be the physical space; it is an affine Euclidean three-dimensional space. Let $G_{1}$ be the group of Euclidean displacements of $E$, i.e., the group of affine Euclidean orientation-preserving mappings of $E$ onto itself. As a reference configuration, we choose a particular position $P_{0}$ of the solid body in $E$. Then for any other position $P$ of the body in $E$, there is a unique $g \in G_{1}$ such that $g P_{0}=P$; conversely, for any $g \in G_{1}, g P_{0}$ is a position of the body in $E$.

Therefore, the group $G_{1}$ can be considered as the configuration space of the system. The space of kinematical states is the tangent bundle $T G_{1}$, and the phase space the cotangent bundle $T^{*} G_{1}$. 
In order to be able to consider $E$ as a vector space, let us choose as origin an arbitrary point $O \in E$. Then the group $G_{1}$ can be identified with the semi-direct product $E \times \mathbf{S O}(E)$, the first factor being the subgroup of translations of $E$, and the second the stabilizer of $O$ in $G_{1}$, i.e., the subgroup of rotations of $E$ around its origin $O$. An element of $G_{1}$ is a pair $(a, g)$, with $a \in E, g \in \mathbf{S O}(E)$, and the mapping of $E$ onto itself which corresponds to that element is

$$
x \mapsto a+g x .
$$

The composition law of $G_{1}$ is

$$
\left(a_{1}, g_{1}\right)\left(a_{2}, g_{2}\right)=\left(a_{1}+g_{1} a_{2}, g_{1} g_{2}\right) .
$$

The Lie algebra $\mathscr{G}_{1}$ of $G_{1}$ is the semi-direct product $E \times \mathbf{s o}(E)$. The first factor $E$ is the Lie algebra of the subgroup of translations of $E$, and the $\operatorname{second} \operatorname{so}(E)$ the Lie algebra of the subgroup of rotations of $E$ around its origin. The bracket in $\mathscr{G}_{1}=E \times \mathbf{s o}(E)$ is given by

$$
\left[\left(a_{1}, X_{1}\right),\left(a_{2}, X_{2}\right)\right]=\left(X_{1} a_{2}-X_{2} a_{1},\left[X_{1}, X_{2}\right]\right),
$$

where, in the right-hand side, $X_{1}$ and $X_{2} \in \mathbf{s o}(E)$ are considered as linear endomorphisms of $E$.

The kinetic energy of the system is

$$
T(w)=\frac{1}{2} K(w, w), \quad w \in T G_{1},
$$

where $K$ is a left-invariant Riemannian metric on $G_{1}$. The potential energy is

$$
U(a, g)=m \gamma\left(a+g C \mid e_{3}\right), \quad(a, g) \in G_{1},
$$

where $(\mid)$ is the scalar product in $E, C$ the position in $E$ of the center of mass of the body when it is in the reference position $P_{0}, e_{3}$ the unit vertical vector pointing upwards, $m$ the total mass of the body and $\gamma$ the gravity acceleration. The Lagrangian of the system is therefore

$$
L(w)=\frac{1}{2} K(w, w)-U(p(w))
$$

where $p: T G_{1} \rightarrow G_{1}$ is the canonical projection.

By left translation, the tangent bundle $T G_{1}$ can be identified with $G_{1} \times \mathscr{G}_{1}$. Since $G_{1}$ and $\mathscr{G}_{1}$ are themselves identified with $E \times \mathbf{S O}(E)$ and $E \times \mathbf{s o}(E)$, respectively, the space of kinematical states is $E \times \mathbf{S O}(E) \times E \times \mathbf{S o}(E)$. A kinematical state will therefore be denoted by $(a, g, v, X)$, with $a \in E, g \in \mathbf{S O}(E), v \in E, X \in \mathbf{s o}(E)$. We observe that $v$ and $X$ can be easily identified, respectively, with the velocity of the point of the body which is situated at the origin when the body is in its reference position, and with the angular velocity of the motion of the body around that point, both expressed in a frame attached to the body.

Let us now choose the reference position $P_{0}$ of the solid body in such a way that when it is in that position, its center of mass is at the origin $O$ of $E$. Then by König's theorem, the kinetic energy splits into a sum

$$
T(a, g, v, X)=\frac{1}{2} m(v \mid v)+\frac{1}{2} I(X, X) .
$$


The first term of that sum, $(1 / 2) m(v \mid v)$, is the kinetic energy of the translation motion of the center of mass of the body. The second, $(1 / 2) I(X, X)$, is the kinetic energy of the rotation of the body around its center of mass. The bilinear form $I$ on the Lie algebra so $(E)$ is symmetric positive definite; it is called the inertia form of the body at its center of mass.

The phase space $T^{*} G_{1}$ can be identified with the product $E \times \mathbf{S O}(E) \times E^{*} \times$ $(\mathbf{s o}(E))^{*}$. An element of that space will be denoted by $(a, g, \Pi, M)$, where $a \in$ $E, g \in \mathbf{S O}(E), \Pi \in E^{*}, M \in(\mathbf{s o}(E))^{*}$. It can be seen easily that $\Pi$ is the linear momentum of the motion of the center of mass of the body, and $M$ the angular momentum of the motion of the body around its center of mass, both expressed in a frame attached to the body.

The Legendre transformation $\mathscr{L}: T G_{1} \rightarrow T^{*} G_{1}$ is the map

$$
(a, g, v, X) \mapsto(a, g, \Pi, M), \quad \text { with } \Pi=\mu(v), M=I(X),
$$

where $\mu: E \rightarrow E^{*}$ and $I: \operatorname{so}(E) \rightarrow(\operatorname{so}(E))^{*}$ are the isomorphisms defined by

$$
\langle\mu(v), w\rangle=m(v \mid w), \quad v \text { and } w \in E, \quad\langle I(X), Y\rangle=I(X, Y), \quad X \text { and } Y \in \mathbf{s o}(E) .
$$

The Hamiltonian $H$ of the system can be written as

$$
H(a, g, \Pi, M)=\frac{1}{2}\left\langle\Pi, \mu^{-1}(\Pi)\right\rangle+\frac{1}{2}\left\langle M, I^{-1}(M)\right\rangle+\gamma\left\langle\mu(a), e_{3}\right\rangle .
$$

4.2. The Constraint Submanifold and the Projection Bundle. When the solid body is in its reference position $P_{0}$, let $\Sigma$ be the surface which bounds that body, considered as a 2-dimensional submanifold of $E$. We recall that the Gauss map, defined on $\Sigma$, with values in the unit sphere $S^{2}$ of $E$, associates with each point of $\Sigma$ the unit vector normal to $\Sigma$ at that point, oriented outwards. Since $\Sigma$ is smooth and strictly convex, the Gauss map is a diffeomorphism. We will denote by $\Gamma: S^{2} \rightarrow \Sigma$ the inverse of the Gauss map.

We assume now that the origin $O$ has been chosen in the horizontal solid plane $F$ on which the moving body is rolling. The constraint submanifold $C$ is the set of kinematical states $(a, g, v, X) \in E \times \mathbf{S} \mathbf{O}(E) \times \mathbf{S o}(E)$ which satisfy the following two conditions:

$$
\begin{gathered}
\left(a \mid e_{3}\right)=\left(\Gamma \circ g^{-1}\left(-e_{3}\right) \mid g^{-1}\left(-e_{3}\right)\right), \\
v+X \circ \Gamma \circ g^{-1}\left(-e_{3}\right)=0 .
\end{gathered}
$$

Equation (9) states that the lowest point of the body is in the horizontal plane $F$. Equation (10) states that the velocity of a material point of the moving body vanishes when that point comes into contact with the horizontal plane $F$ on which the body is rolling.

It is easy to verify that the constraint is regular in the sense of Definition 2.5. The submanifold denoted by $Q_{1}$ in that definition is the subset of elements $(a, g) \in$ $E \times \mathbf{S O}(E)$ which satisfy $(9)$. We observe that $Q_{1}$ and the constraint submanifold $C$ can be identified with $F \times \mathbf{S O}(E)$ and $F \times \mathbf{S O}(E) \times \mathbf{S o}(E)$, respectively, since (9) and (10) determine $\left(a \mid e_{3}\right)$ in terms of $g$, and $v$ in terms of $g$ and $X$. The variable which runs over the factor $F$ is the orthogonal projection on $F$ of the center of mass of the moving body. 
The Hamiltonian constraint submanifold $D$ is the subset of elements $(a, g, \Pi, M)$ $\in E \times \mathbf{S O}(E) \times E^{*} \times(\mathbf{s o}(E))^{*}$ which satisfy (9) and

$$
\Pi+\mu \circ\left(I^{-1} M\right) \circ \Gamma \circ g^{-1}\left(-e_{3}\right)=0 \text {. }
$$

It can be identified with $F \times \mathbf{S O}(E) \times(\mathbf{s o}(E))^{*}$.

For each $g \in \mathbf{S O}(E)$, we will denote by $\varphi_{g}: E^{*} \rightarrow(\mathbf{s o}(E))^{*}$ the linear map defined by

$$
\left\langle\varphi_{g} \Pi^{\prime}, Y\right\rangle=\left\langle\Pi^{\prime}, Y \circ g^{-1}\left(-e_{3}\right)\right\rangle, \quad \Pi^{\prime} \in E^{*}, \quad Y \in \mathbf{s o}(E) .
$$

Then it is easy to see that the fiber of the projection bundle over a point $(a, g, \Pi, M)$ of $D$ is the set of vectors tangent at that point to $E \times \mathbf{S O}(E) \times E^{*} \times(\operatorname{so}(E))^{*}$, whose projections on the first two factors $E$ and $\mathbf{S O}(E)$ vanish, and whose projections $\Pi^{\prime}$ on $E^{*}$ and $M^{\prime}$ on $(\operatorname{so}(E))^{*}$ satisfy

$$
M^{\prime}=\varphi_{g} \Pi^{\prime}
$$

4.3. The Evolution Equations for the Constrained System. By using well known formulas for the Liouville 1-form on $T^{*} G_{1}$ and its differential (which may be found for example in [15]), we obtain the evolution equations of the constrained mechanical system:

$$
\left\{\begin{array}{l}
\frac{d a}{d t}=g \mu^{-1} \Pi \\
\frac{d g}{d t}=g I^{-1} M \\
\frac{d \Pi}{d t}={ }^{t}\left(I^{-1} M\right) \Pi-\gamma \mu g^{-1}\left(e_{3}\right)+\Pi^{\prime} \\
\frac{d M}{d t}=-\operatorname{ad}_{I^{-1} M}^{*} M+\varphi_{g} \Pi^{\prime}
\end{array}\right.
$$

In the third equation above, ${ }^{t}\left(I^{-1} M\right)$ is the linear endomorphism of $E^{*}$ transpose of $I^{-1} M \in \mathbf{s o}(E)$, considered as a linear endomorphism of $E$. We recall our sign convention about the coadjoint action, used in the fourth equation above:

$$
\left\langle\operatorname{ad}_{X}^{*} M, Y\right\rangle=\left\langle M,-\operatorname{ad}_{X} Y\right\rangle=\langle M,[Y, X]\rangle, M \in(\operatorname{so}(E))^{*}, X \text { and } Y \in \mathbf{s o}(E) .
$$

The element $\Pi^{\prime} \in(\mathbf{s o}(E))^{*}$ must be chosen in such a way that for every $t \in$ $\mathbf{R}, \quad(a(t), g(t), \Pi(t), M(t))$ remains in the Hamiltonian constraint submanifold $D$ defined by Eqs. (9) and (11). We shall say (maybe a little improperly) that $\Pi^{\prime}$ is the constraint force, since it determines that force.

4.4. The Reduced Constrained Mechanical System. The symmetry group $G$ of the system is the subgroup of $G_{1}=E \times \mathbf{S O}(E)$ generated by translations parallel to the horizontal plane $F$ and by rotations around the vertical axis through the origin $O$. Elements of $G$ are pairs $(b, h) \in E \times \mathbf{S O}(E)$ such that

$$
(b \mid e)=0, \quad h e_{3}=e_{3} .
$$

The symmetry group $G$ acts on $G_{1}$ by multiplication on the left,

$$
((b, h),(a, g)) \mapsto(b+h a, h g) .
$$

It acts on the phase space $T^{*} G_{1}$ by the canonical lift of that action to the cotangent bundle. If we identify $T^{*} G_{1}$ with $E \times \mathbf{S O}(E) \times E^{*} \times(\mathbf{s o}(E))^{*}$ by left translations, 
that canonical lift is given by

$$
((b, h),(a, g, \Pi, M)) \mapsto(b+h a, h g, \Pi, M) .
$$

The set $\widehat{M}$ of orbits of that action can be identified with $\mathbf{R} \times S^{2} \times E^{*} \times(\mathbf{s o}(E))^{*}$, and the canonical projection of $M=E \times \mathbf{S O}(E) \times E^{*} \times(\mathbf{s o}(E))^{*}$ onto $\widehat{M}$ is

$$
(a, g, \Pi, M) \mapsto\left(z=\left(a \mid e_{3}\right), n=g^{-1}\left(-e_{3}\right), \Pi, M\right) .
$$

The Poisson structure on $\widehat{M}$ for which the canonical projection is a Poisson map is easily derived from the formulas for the symplectic 2-form on $T^{*} G_{1}$. The Poisson bracket of two smooth functions $F_{1}$ and $F_{2}$ at a point $(z, n, \Pi, M)$ of $\widehat{M}$ is

$$
\begin{aligned}
\left\{F_{1}, F_{2}\right\}= & \left(d_{\Pi} F_{1} \mid e_{3}\right) \frac{\partial F_{2}}{\partial z}-\left(d_{\Pi} F_{2} \mid e_{3}\right) \frac{\partial F_{1}}{\partial z} \\
& +\left\langle d_{n} F_{1},\left(d_{M} F_{2}\right) n\right\rangle-\left\langle d_{n} F_{2},\left(d_{M} F_{1}\right) n\right\rangle \\
& +\left\langle\Pi,\left(d_{M} F_{1}\right)\left(d_{\Pi} F_{2}\right)-\left(d_{M} F_{2}\right)\left(d_{\Pi} F_{1}\right)\right\rangle \\
& +\left\langle M,\left[d_{M} F_{1}, d_{M} F_{2}\right]\right\rangle .
\end{aligned}
$$

In this formula, $d_{n} F_{i} \in T_{n}^{*} S^{2}, d_{\Pi} F_{l} \in E, d_{M} F_{i} \in \mathbf{s o}(E)$ are the partial differentials of $F_{l}(i=1$ or 2$)$, at point $(z, n, \Pi, M)$, with respect to the variables $n \in S^{2}, \Pi \in$ $E^{*}, M \in(\mathbf{s o}(E))^{*}$, respectively.

The reduced Hamiltonian is

$$
\widehat{H}(z, n, \Pi, M)=\frac{1}{2}\left\langle\Pi, \mu^{-1} \Pi\right\rangle+\frac{1}{2}\left\langle M, I^{-1} M\right\rangle+m \gamma z .
$$

The reduced Hamiltonian constraint submanifold $\widehat{D}$ is the subset of elements $(z, n, \Pi, M)$ in $\widehat{M}$ which satisfy

$$
\begin{gathered}
z=(\Gamma(n) \mid n), \\
\Pi+\mu \circ\left(I^{-1} M\right) \circ \Gamma(n)=0 .
\end{gathered}
$$
by

For each $b \in E$, we will denote by $\psi_{b}: E^{*} \rightarrow(\operatorname{so}(E))^{*}$ the linear map defined

$$
\left\langle\psi_{b} \Pi^{\prime}, Y\right\rangle=\left\langle\Pi^{\prime}, Y b\right\rangle, \quad \Pi^{\prime} \in E^{*}, \quad Y \in \mathbf{s o}(E) .
$$

Then the fiber of the reduced projection bundle $\widehat{W}$ over a point $(z, n, \Pi, M)$ of $\widehat{D}$, is the set of vectors tangent to $\widehat{M}$ at that point whose projections on the first two factors $\mathbf{R}$ and $S^{2}$ vanish, and whose projections $\Pi^{\prime}$ on the third factor $E^{*}$, and $M^{\prime}$ on the fourth factor $(\mathbf{s o}(E))^{*}$, satisfy

$$
M^{\prime}=\psi_{\Gamma(n)} \Pi^{\prime} .
$$

The evolution equations for the reduced constrained Hamiltonian system are

$$
\left\{\begin{array}{l}
\frac{d z}{d t}=-\left(\mu^{-1} \Pi \mid n\right) \\
\frac{d n}{d t}=-\left(I^{-1} M\right) n \\
\frac{d \Pi}{d t}={ }^{t}\left(I^{-1} M\right) \Pi+\gamma \mu n+\Pi^{\prime} \\
\frac{d M}{d t}=-\operatorname{ad}_{I^{-1} M}^{*} M+\psi_{\Gamma(n)} \Pi^{\prime}
\end{array}\right.
$$


The constraint force $\Pi^{\prime}$ must be chosen in such a way that for all $t \in \mathbf{R},(z(t), n(t)$, $\Pi(t), M(t))$ lies in the reduced constraint submanifold $\widehat{D}$, defined by Eqs. (11) and $(12)$.

We observe that the first Eq. (15), which expresses $d z / d t$, is in fact a consequence of the other three Eqs. (15) and of Eqs. (12) and (13), which define $\widehat{D}$. Therefore the really useful equations which govern the time evolution of the reduced system are (12), (13), and the last three Eqs. (15).

We observe also that the reduced Hamiltonian $\widehat{H}$ is a first integral of the evolution equations.

\subsection{Elimination of the Constraint Force. Let us introduce}

$$
P=M-\psi_{\Gamma(n)} \Pi \text {. }
$$

This new variable, defined on the reduced Hamiltonian constraint submanifold, has a clear mechanical meaning: it is the angular momentum of the solid body at its contact point with the horizontal plane on which it is rolling, expressed in a frame attached to the body.

By using Eqs. (13) and (15), we obtain

$$
\frac{d P}{d t}=-\operatorname{ad}_{I^{-1} M}^{*} P-\gamma \psi_{\Gamma(n)} \circ \mu(n)-\psi_{T_{n} \Gamma\left(\left(I^{-1} M\right) n\right)} \circ \mu \circ\left(I^{-1} M\right) \circ \Gamma(n) .
$$

We have denoted by $T_{n} \Gamma: T_{n} S^{2} \rightarrow T_{\Gamma(n)} E \equiv E$ the tangent map to the inverse $\Gamma$ of the Gauss map at $n \in S^{2}$. We have used the identity, which follows easily from the definition (14) of $\psi$,

$$
\psi_{\Gamma(n)}\left({ }^{t}\left(I^{-1} M\right) \Pi\right)=-\operatorname{ad}_{I^{-1} M}^{*} \psi_{\Gamma(n)} \Pi-\psi_{\left(I^{-1} M\right) \circ \Gamma(n)} \Pi,
$$

and the fact, which follows from Eq. (13) and the skew-symmetry of elements of $\operatorname{so}(E)$, that

$$
\psi_{\left(I^{-1} M\right) \circ \Gamma(n)} \Pi=\psi_{\left(I^{-1} M\right) \circ \Gamma(n)}\left(-\mu \circ\left(I^{-1} M\right) \circ \Gamma(n)\right)=0 .
$$

Now we use the fact that the Euclidean vector space $E$ is of dimension 3. We know that once a particular orientation of $E$ is chosen, we can define on that space a composition law, called the vector product and denoted by $(x, y) \mapsto x \times y$. We recall that for any positively oriented orthonormal basis $\left(e_{1}, e_{2}, e_{3}\right)$ of $E$, we have

$$
e_{1} \times e_{2}=e_{3}, \quad e_{2} \times e_{3}=e_{1}, \quad e_{3} \times e_{1}=e_{2},
$$

and that $E$, when equipped with that composition law, is a Lie algebra. We recall the well known formulae which relate the vector and scalar products on $E$ :

$$
\begin{aligned}
(x \times y \mid z) & =(x \mid y \times z), \\
x \times(y \times z) & =(x \mid z) y-(x \mid y) z,
\end{aligned} \quad x, y \text { and } z \in E .
$$

We know also that the map $\zeta: \mathbf{s o}(E) \rightarrow E$, defined by

$$
\left(\zeta^{-1} x\right) y=x \times y, \quad x \text { and } y \in E,
$$

is a Lie algebra homomorphism. 
Using the above formulae and Eq. (14), we obtain for any $b \in E$ a new formula for the linear map $\psi_{b}: E^{*} \rightarrow(\operatorname{so}(E))^{*}$ :

$$
\psi_{b}=\operatorname{ad}_{\zeta^{-1} b}^{*}{ }^{t} \zeta
$$

Then, for any $Y \in \mathbf{s o}(E)$, we deduce from Eqs. (13) and (16):

$$
\begin{aligned}
\langle P, Y\rangle= & \langle M, Y\rangle+\left\langle\operatorname{ad}_{\zeta-1}^{*} \Gamma(n){ }^{t} \zeta \circ \mu \circ\left(I^{-1} M\right) \circ \Gamma(n), Y\right\rangle \\
= & \langle M, Y\rangle-m\left\langle\zeta\left(I^{-1} M\right) \times \Gamma(n) \mid \Gamma(n) \times \zeta(Y)\right\rangle \\
= & \langle M, Y\rangle+m\left(\Gamma(n) \times\left(\zeta\left(I^{-1} M\right) \times \Gamma(n)\right) \mid \zeta(Y)\right) \\
= & \langle M, Y\rangle+(\Gamma(n) \mid \Gamma(n))\left\langle{ }^{t} \zeta \circ \mu \circ \zeta\left(I^{-1} M\right), Y\right\rangle \\
& -\left(\Gamma(n) \mid \zeta\left(I^{-1} M\right)\right)\left\langle{ }^{t} \zeta \circ \mu \circ \Gamma(n), Y\right\rangle .
\end{aligned}
$$

Therefore we have

$$
P=J(n)\left(I^{-1} M\right)-\left(\Gamma(n) \mid \zeta\left(I^{-1} M\right)\right)^{t} \zeta \circ \mu \circ \Gamma(n),
$$

where $J(n): \mathbf{s o}(E) \rightarrow(\operatorname{so}(E))^{*}$ is the linear map

$$
J(n)=I+(\Gamma(n) \mid \Gamma(n))^{t} \zeta \circ \mu \circ \zeta .
$$

For any $Y$ and $Z \in \mathbf{s o}(E)$, we have

$$
\langle J(n) Y, Z\rangle=\langle I Y, Z\rangle+m(\Gamma(n) \mid \Gamma(n))(\zeta Y \mid \zeta Z),
$$

which proves that $J(n)$ is symmetric positive definite (with respect to the scalar product on $\operatorname{so}(E)$ for which $\zeta$ is an isometry). Therefore, $J(n)$ is invertible, and we have

$$
J(n)^{-1}(P)=I^{-1} M-\left(\Gamma(n) \mid \zeta\left(I^{-1} M\right)\right) J(n)^{-1} \circ{ }^{t} \zeta \circ \mu \circ \Gamma(n) .
$$

By applying $\zeta$ and taking the scalar product with $\Gamma(n)$, we obtain

$$
\left(\Gamma(n) \mid \zeta \circ J(n)^{-1}(P)\right)=\left(\Gamma(n) \mid \zeta\left(I^{-1} M\right)\right)\left(1-\left(\Gamma(n) \mid \zeta \circ J(n)^{-1} \circ{ }^{t} \zeta \circ \mu \circ \Gamma(n)\right)\right) .
$$

Using the isomorphism $\zeta$ and the Euclidean structure of $E$, we can identify $E$, so $(E)$ and their duals. Let $x_{i}$ be the components of $\Gamma(n)$ in an orthonormal basis of $E$ in which $I$ is diagonal, with diagonal components $I_{i}, i=1,2,3$. In that basis, $J(n)$ is also diagonal, with diagonal components

$$
J_{i}=I_{i}+m\left(x_{1}^{2}+x_{2}^{2}+x_{3}^{2}\right), \quad i=1,2,3 .
$$

The components of $\zeta \circ J(n)^{-1} \circ{ }^{t} \zeta \circ \mu \circ \Gamma(n)$ are:

$$
\frac{m x_{l}}{J_{l}}=\frac{m x_{i}}{I_{l}+m\left(x_{1}^{2}+x_{2}^{2}+x_{3}^{2}\right)},
$$

and we have

$$
\begin{aligned}
& 1-\left(\Gamma(n) \mid \zeta \circ J(n)^{-1} \circ{ }^{t} \zeta \circ \mu \circ \Gamma(n)\right)=1-m \sum_{i=1}^{3} \frac{x_{i}^{2}}{I_{i}+m\left(x_{1}^{2}+x_{2}^{2}+x_{3}^{2}\right)} \\
& \geqq 1-\frac{m\left(x_{1}^{2}+x_{2}^{2}+x_{3}^{2}\right)}{\inf \left(I_{1}, I_{2}, I_{3}\right)+m\left(x_{1}^{2}+x_{2}^{2}+x_{3}^{2}\right)}>0,
\end{aligned}
$$


since $\inf \left(I_{1}, I_{2}, I_{3}\right)>0$. We may therefore write

$$
\left(\Gamma(n) \mid \zeta\left(I^{-1} M\right)\right)=\frac{\left(\Gamma(n) \mid \zeta \circ J(n)^{-1}(P)\right)}{1-\left(\Gamma(n) \mid \zeta \circ J(n)^{-1} \circ t \zeta \circ \mu \circ \Gamma(n)\right)},
$$

and, using (19),

$$
I^{-1} M=J(n)^{-1}(P)-\frac{\left(\Gamma(n) \mid \zeta \circ J(n)^{-1}(P)\right)}{1-\left(\Gamma(n) \mid \zeta \circ J(n)^{-1} \circ^{t} \zeta \circ \mu \circ \Gamma(n)\right)} J(n)^{-1} \circ{ }^{t} \zeta \circ \mu \circ \Gamma(n) .
$$

The above equation yields $I^{-1} M$ in terms of $P$ and $n$ only. Let us replace $I^{-1} M$ by that expression in the second Eq. (15) and in Eq. (17). We obtain a differential equation for $(n, P) \in S^{2} \times(\mathbf{s o}(E))$, which is the evolution equation for the reduced constrained mechanical system.

\section{Two Simple Examples}

5.1. First example: Description of the System. The configuration space of the system is the cylinder $Q=\mathbf{R} \times S^{1}$. We denote by $(x, \theta)$ and $(x, \theta, \dot{x}, \dot{\theta})$ the usual local coordinates on $Q$ and $T Q$, respectively. Observe that $\theta \in S^{1}=\mathbf{R} / 2 \pi \mathbf{Z}$ is an angle rather than a real.

We choose for the constraint submanifold

$$
C=\{(x, \theta, \dot{x}, \dot{\theta}) \in T Q \mid \dot{x}+x \dot{\theta}=0\} .
$$

It is a rank 1, therefore integrable sub-bundle of $T Q$ (the constraint is holonomic), and the leaves of the foliation of $Q$ defined by $C$ are the images of immersions of $\mathbf{R}$ into $Q$ of the form

$$
\theta \mapsto\left(c e^{-\theta}, \theta \text { modulo } 2 \pi\right),
$$

where $c$ is a constant. The leaf $c=0$ is a circle $S^{1}$, and all the other leaves are (for their own manifold structure) diffeomorphic to $\mathbf{R}$.

For the Lagrangian, we choose

$$
L(x, \theta, \dot{x}, \dot{\theta})=\frac{1}{2}\left(\dot{x}^{2}+\dot{\theta}^{2}\right) .
$$

The mechanical system $(Q, L, C)$ has a very simple mechanical interpretation: it describes the motion of a material point of unit mass, constrained on a cylinder on which a system of grooves imposes the relation $\dot{x}+x \dot{\theta}=0$ between the two components of the velocity. Alternatively, the system can also be physically realized by a skate moving on a cylinder, with a mechanism which imposes the angle made by the blade of the skate with the axis of the cylinder, as a function of the coordinate $x$ of the skate along that axis.

We denote by $\left(x, \theta, p_{x}, p_{\theta}\right)$ the coordinates on the phase space $T^{*} Q$. The Legendre transformation is

$$
(x, \theta, \dot{x}, \dot{\theta}) \mapsto\left(x, \theta, p_{x}=\dot{x}, p_{0}=\dot{\theta}\right),
$$

and the Hamiltonian of the system is

$$
H\left(x, \theta, p_{x}, p_{\theta}\right)=\frac{1}{2}\left(p_{x}^{2}+p_{\theta}^{2}\right) .
$$


The Hamiltonian constraint submanifold is

$$
D=\left\{\left(x, \theta, p_{x}, p_{0}\right) \in T^{*} Q \mid p_{x}+x p_{0}=0\right\},
$$

and the projection bundle $W$ is generated by the vector field along $D$

$$
\frac{\partial}{\partial p_{x}}+x \frac{\partial}{\partial p_{0}}
$$

The Hamiltonian vector field $X_{H}$ and the constrained Hamiltonian vector field $X_{D}$ are, respectively,

$$
X_{H}=p_{x} \frac{\partial}{\partial x}+p_{0} \frac{\partial}{\partial \theta}, \quad X_{D}=p_{x} \frac{\partial}{\partial x}+p_{0} \frac{\partial}{\partial \theta}+\lambda\left(\frac{\partial}{\partial p_{x}}+x \frac{\partial}{\partial p_{0}}\right)
$$

where $\lambda$ must be chosen so that $X_{D}$ be tangent to $D$. We obtain

$$
\lambda=-\frac{p_{x} p_{\theta}}{1+x^{2}} .
$$

The equations of motion are therefore

$$
\left\{\begin{array} { l } 
{ \frac { d x } { d t } = p _ { x } , } \\
{ \frac { d \theta } { d t } = p _ { \theta } , }
\end{array} \quad \left\{\begin{array}{l}
\frac{d p_{x}}{d t}=-\frac{p_{x} p_{\theta}}{1+x^{2}}, \\
\frac{d p_{\theta}}{d t}=-\frac{x p_{x} p_{\theta}}{1+x^{2}} .
\end{array}\right.\right.
$$

We must also add to the system the constraint equation

$$
p_{x}+x p_{0}=0
$$

and choose Cauchy data which satisfy that equation. The corresponding solution of (20) will then satisfy (21) for all $t$.

5.2. First example: The $S^{1}$ action and the Reduced System. We consider the action $\Phi: S^{1} \times Q \rightarrow Q$,

$$
(\alpha,(x, \theta)) \mapsto(x, \alpha+\theta) .
$$

Its canonical lifts to $T Q$ and $T^{*} Q$ are, respectively,

$$
(\alpha,(x, \theta, \dot{x}, \dot{\theta})) \mapsto(x, \alpha+\theta, \dot{x}, \dot{\theta})
$$

and

$$
\left(\alpha,\left(x, \theta, p_{x}, p_{0}\right)\right) \mapsto\left(x, \alpha+\theta, p_{x}, p_{0}\right) .
$$

The constraint submanifold $C$ and the hamiltonian constraint submanifold $D$ are invariant under these actions, as well as the foliation of $Q$ defined by $C$. However, the leaves of that foliation are individually not invariant in general.

The quotient Poisson manifold $\widehat{M}=T^{*} Q / S^{1}$ is diffeomorphic to $\mathbf{R}^{3}$ (coordinates $\left.x, p_{x}, p_{0}\right)$, with the Poisson tensor

$$
\widehat{\Lambda}=\frac{\partial}{\partial p_{x}} \wedge \frac{\partial}{\partial x} .
$$


The expression of the reduced Hamiltonian $\widehat{H}$ coincides with that of $H$, since $H$ does not depend on $\theta$. The reduced hamiltonian constraint submanifold $\widehat{D}$ is

$$
\widehat{D}=\left\{\left(x, p_{x}, p_{0}\right) \in \widehat{M} \mid p_{x}+x p_{\theta}=0\right\} .
$$

It can be identified with $\mathbf{R}^{2}$, with $x$ and $p_{\theta}$ as coordinates. The reduced projection bundle $\widehat{W}$ is generated by the vector field along $\widehat{D}$,

$$
\frac{\partial}{\partial p_{x}}+x \frac{\partial}{\partial p_{\theta}}
$$

With $x$ and $p_{\theta}$ as coordinates on $\widehat{D}$, the reduced equations of motion are

$$
\left\{\begin{aligned}
\frac{d x}{d t} & =p_{x}, \\
\frac{d p_{0}}{d t} & =\frac{x^{2} p_{0}^{2}}{1+x^{2}} .
\end{aligned}\right.
$$

Since $\widehat{H}$ is a first integral, each integral curve satisfies

$$
\left(1+x^{2}\right) p_{0}^{2}=\text { constant }
$$

All the points of the first coordinate axis $\left(x, p_{\theta}=0\right)$, including the origin $(x=$ $\left.0, p_{\theta}=0\right)$ are equilibria of the reduced system, which correspond to true equilibria of the nonreduced system. All the points of the second axis $\left(x=0, p_{\theta} \neq 0\right)$, except the origin, are equilibria of the reduced system which correspond to relative equilibria (stationary motions) of the nonreduced system. It is easy to see that in $\widehat{D}$, the half axis $\left\{\left(x=0, p_{\theta}>0\right)\right\}$ is attractive (its attractive basin is the half plane $\left.p_{\theta}>0\right)$, and that the other half axis $\left\{\left(x=0, p_{\theta}<0\right)\right\}$ is repulsive.

5.3. Another Example. We indicate here a simple example of constrained Hamiltonian system in the sense of Definition 3.1, with a stable and attractive equilibrium. We do not use here the reduction procedure: our example should be considered as an already reduced system. The reader is referred to A. Weinstein [41] for a general discussion of stability of equilibria in Poisson Hamiltonian systems.

The phase space is $\mathbf{R}^{3}$ (coordinates $x, y, z$ ), with the Lie-Poisson structure of $(\mathbf{s o}(3))^{*}$,

$$
\Lambda=z \frac{\partial}{\partial x} \wedge \frac{\partial}{\partial y}+x \frac{\partial}{\partial y} \wedge \frac{\partial}{\partial z}+y \frac{\partial}{\partial z} \wedge \frac{\partial}{\partial x} .
$$

The Hamiltonian of the system is the linear form

$$
H(x, y, z)=\alpha x+\beta y+\gamma z, \alpha, \beta \text { and } \gamma \in \mathbf{R} \text {, constants . }
$$

The Hamiltonian constraint submanifold $D$ is the plane $z=0$. The projection bundle $W$ is generated by the vector field along $D$

$$
\frac{\partial}{\partial z}+a \frac{\partial}{\partial x}, \quad a \in \mathbf{R} \text { constant }
$$

For the constrained Hamiltonian vector field $X_{D}$ we obtain

$$
X_{D}=(-a \beta x+(\gamma+a \alpha) y) \frac{\partial}{\partial x}-\gamma x \frac{\partial}{\partial y} .
$$


It is easy to see that if the constants $a, \alpha$ and $\gamma$ satisfy

$$
\gamma(a \alpha+\gamma)>0
$$

the origin is a stable and attractive equilibrium of $X_{D}$.

Acknowledgements. Several years ago, Rudolf Schmid first spoke to me about the curious behaviour of celtic stones. More recently J.J. Duistermaat suggested that it would be interesting to explain the attractiveness of their stationary motions by geometric means and indicated to me Ref. [9, 10 and 17]. I have had very stimulating discussions with Pierre Dazord about his work [7], with Richard Cushman who indicated to me Ref. [4], and with Paulette Libermann, who suggested to me several important improvements. Marco Favretti indicated to me Ref. [33]. Jean-Pierre Marco read carefully preliminary versions of this work and drew my attention to the fact that attractive relative equilibria can exist in mechanical systems with holonomic constraints. Jean-Pierre Françoise, Daniel Leborgne, Izu Vaisman and Pierre Molino encouraged me by their interest in this work and gave me the opportunity to speak about Mechanics in their seminars. I address my warmest thanks to them all.

\section{References}

1. Abraham, R., Marsden, J.E., Foundations of mechanics. Reading, MA: Benjamin, 1978

2. Albert, C.: Le théorème de réduction de Marsden-Weinstein en géométrie cosymplectique et de contact. J. Geom. and Phys. 6, 627-649 (1989)

3. Arnold, V.I., Kozlov, V.V., Neishtadt, A.I.: Mathematical aspects of classical and celestial mechanics. In: Dynamical systems III, Arnold, V.I. (ed.), Berlin, Heidelberg, New York: Springer, 1988

4. Bates, L., Sniatycki, J.: Nonholonomic reduction. Rep. Math. Phys. 32, 99-115 (1991)

5. Benenti, S.: Geometrical aspects of the dynamics of non-holonomic systems. Journées relativistes, Chambéry, 14-16 May 1987

6. Cardin, F. and Zanzotto, G.: On constrained mechanical systems: d'Alembert's and Gauss' principles. J. Math. Phys. 30, 7, 1473-1479 (1989)

7. Dazord, P.: Mécanique hamiltonienne en présence de contraintes. Illinois J. Math. 38, 1, 148175 (1994)

8. Godbillon, C.: Géométrie différentielle et mécanique analytique. Paris: Hermann, 1969

9. Karapetian, A.V.: On the problem of steady motions stability of nonholonomic systems. Prikl. Matem. Mekhan. 44, 3, 418-426 (1980) (in Russian). English translation: PMM USSR 44, 295-300 (1980)

10. Karapetian, A.V.: On realizing nonholonomic constraints by viscous friction forces and celtic stones stability. Prikl. Matem. Mekhan. 45, 1, 42-51 (1981) (in Russian). English translation: PMM USSR 45, 30-36 (1981)

11. Koiller, J.: Reduction of some classical non-holonomic systems with symmetry. Arch. Rational Mech. Anal. 118, 133-148 (1992)

12. Krishnaprasad, P.S.: Eulerian many-body problems. In: Dynamics and control of multi-body systems. Marsden, J.E., Krishnaprasad, P.S., Simo, J.C. (eds.), Contemporary Mathematics 97, Providence, RI: American Mathematical Society, 1989, pp. 187-208

13. Krishnaprasad, P.S., Yang, R.: Geometric phase, anholonomy, and optimal movement. Proc. of the 1991 IEEE International conference on Robotics and Automation 3, 2185-2189 (1991)

14. Kummer, M.: On the construction of the reduced phase space of a Hamiltonian system with symmetry. Indiana Univ. Math. J. 30, 2, 281-291 (1981)

15. Libermann, P., Marle, C.-M.: Symplectic geometry and analytical mechanics. Dordrecht: D. Reidel Publishing Company, 1987

16. Lichnerowicz, A.: Les variétés de Poisson et leurs algèbres de Lie associées. J. Differ. Geom. 12, 253-300 (1977)

17. Markeev, A.P.: On the dynamics of a solid on an absolutely rough plane. Prikl. Matem. Mekhan. 47, 4, 575-582 (1983) (in Russian). English translation. PMM USSR 47, 4, 473478 (1983) 
18. Marle, C.-M.: Sur la géométrie des systèmes mécaniques à liaisons actives. C. R. Acad. Sci. Paris 311, I, 839-845 (1990)

19. Marle, C.-M.: Géométrie des systèmes mécaniques à liaisons actives. In: Symplectic geometry and mathematical physics, Donato, P., Duval, C., Elhadad, J., Tuynman, G.M. (eds.), Boston: Birkhäuser, 1991, pp. 260-287

20. Marsden, J.E.: Lectures on mechanics. London Math. Soc. Lect. Notes Series 174, Cambridge: Cambridge University Press, 1992

21. Marsden, J.E. and Ratiu, T.S.: Reduction of Poisson manifolds. Lett. Math. Phys. 11, 161-170 (1986)

22. Marsden, J., Weinstein, A.: Reduction of symplectic manifolds with symmetry. Rep. Math. Phys. 5, 121-130 (1974)

23. Massa, E., Pagani, E.: Classical dynamics of non-holonomic systems: A geometric approach. Ann. Inst. Henri Poincaré, Physique Théor. 55, 1, 511-544 (1991)

24. Meyer, K.R.: Symmetry and integrals in mechanics. In: Dynamical systems, Peixoto, M. (ed.), New York: Academic Press, 1973, pp. 259-273

25. Mikami, K.: Reduction of local Lie algebra structures. Proc. Am. Math. Soc. 105, 3, 686-691 (1989)

26. Montgomery, R., Marsden, J.E., Ratiu, T.: Gauged Lie-Poisson structures. In: Fluids and plasmas: Geometry and dynamics. Contemporary mathematics 28, Providence, RI: Am. Math. Soc., 1984, pp. 101-114

27. Nunes da Costa, J.: Réduction des varietés de Jacobi. C. R. Acad. Sc. Paris 308, I, 101-103 (1989)

28. Pascal, M.: The asymptotic solution of the equations of motion of the celtic stone. Prikl. Matem. Mekhan. 47, 2, 321-329 (1983) (in Russian). English translation: PMM USSR 47, 2 , 269-276 (1984)

29. Souriau, J.-M.: Structure des systèmes dynamiques. Paris: Dunod, 1969

30. Sternberg, S.: On minimal coupling and the symplectic mechanics of a classical particle in the presence of a Yang-Mills field. Proc. Nat. Acad. Sci. USA 74, 5253-5254 (1977)

31. Tulczyjew, W.M.: Sur la différentielle de Lagrange. C.R. Acad. Sc. Paris 280, A, 1295-1298 (1975)

32. Tulczyjew, W.M.: Geometric formulations of physical theories. Napoli, Italy: Bibliopolis, 1989

33. Vershik, A.M.: Classical and non-classical dynamics with constraints. In: Global analysis, Studies and Applications 1, Yu. G. Borisovich and Yu. E. Gliklikh, ed., Lecture Notes in Math. 1108, Berlin: Springer-Verlag, 1984, pp. 278-301

34. Vershik, A.M., Faddeev, L.D.: Lagrangian mechanics in invariant form. Sel. Math. Sov 1, 339-350 (1981)

35. Vershik, A.M., Gershkovich, Y.Ya.: Nonholonomic dynamical systems, geometry of distributions and variational problems. In: Dynamical systems VII, Arnold, V.I. and Novikov, S.P. (eds.), Berlin, Heidelberg, New York: Springer, 1994, pp. 1-81

36. Walker, G.T.: On the curious dynamical properties of celts. Proceedings of the Cambridge Philosophical Society, vol. 8, years 1892-1895, p. 305

37. Walker, G.T.: On a dynamical top. Quart. J. Pure and Appl. Math. 28, 175-184 (1896)

38. Walker, J.: The mysterious "rattleback": A stone that spins in one direction and then reverses. Scientific American 241, 4, 172-184 (1979)

39. Weinstein, A.: A universal phase space for particles in Yang-Mills field. Letters Math. Phys. 2, 417-420 (1978)

40. Weinstein, A.: The local structure of Poisson manifolds. J. Differ. Geom. 18, 523-557 (1983) and 22, 255 (1985)

41. Weinstein, A.: Stability of Poisson-Hamilton equilibria. In: Fluids and plasmas: Geometry and dynamics, Contemporary Mathematics 28, Providence RI: Am. Math. Soc., 1984, pp. 3-13

42. Whittaker, E.T.: A treatise on the analytical dynamics of particles and rigid bodies. Cambridge: Cambridge University Press, 1904 (fourth edition 1937, reissued with Foreword 1988)

43. Woodhouse, N.: Geometric quantization. Oxford: Oxford University Press, 1980

44. Yang, R.: Nonholonomic geometry, Mechanics and Control. Ph.D. Dissertation, University of Maryland, 1992 